\title{
In memoriam Bernard Droz
}

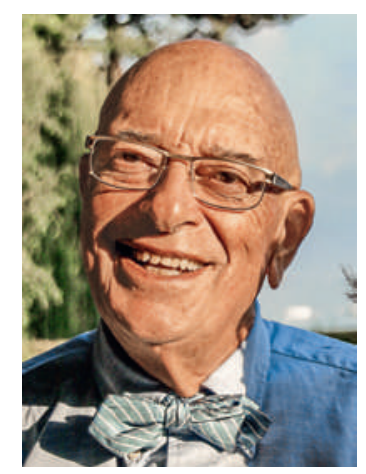

Prof. Dr Bernard Droz

25.3.1930-9.4.2015

Professeur Bernard Droz vient de nous quitter le 9 avril 2015 à l'âge de 85 ans.

Cet éminent scientifique, Professeur honoraire d'histologie et d'embryologie, a été apprécié par de nombreuses volées de futurs médecins. Chercheur et auteur de nombreuses découvertes dans le domaine des neurosciences (fonctionnement de la transmission axonale), il a été appelé à exercer les plus hautes responsabilités dans les institutions responsables de la recherche, Brain Research, ainsi que le CNRS et l'INSERM en France, le Fonds national en Suisse.

Loin d'être un scientifique qui écrase de son aura professorale, c'était au contraire un homme sage, profondément humain, et engagé. Il était actif non seulement dans son domaine spécialisé et dans de multiples sociétés scientifiques, mais également dans les organes de l'Université de Lausanne, au Centre catholique de radio et de télévision, et au groupe des professionnels de santé d'Amnesty International. Jusqu'à ses derniers jours, oubliant son âge, des deuils douloureux et un état de santé miné par plusieurs affections graves, il restait toujours profondément interpellé par l'injustice, la violence et la misère de ses frères humains. Il était toujours disponible pour répondre aux demandes souvent urgentes qui lui étaient faites par Amnesty International et en février de cette année, il écrivait encore: «S'inquiéter, se soucier, tenter d'aider les plus démunis de liberté, de justice et de soins médicaux, n'est ce pas donner un sens à la fin de sa vie?» Quel magnifique message, quel témoignage et quel exemple! (On pense à Stéphane Hessel, auteur de Indignez vous.)

Dr Yves Guisan, spécialiste en chirurgie et médecine générale FMH, ancien conseiller national

Dr J. Molleyres, coordinatrice des Actions Urgentes pour le Groupe des Professionnels de santé 